\title{
Modeling the transmission dynamics of HIV/AIDS epidemics: an introduction and a review
}

\author{
Onoja Matthew Akpa ${ }^{1}$ and Benjamin Agboola Oyejola ${ }^{2}$ \\ ${ }^{1}$ Department of Mathematical Science, Redeemer's University, Redemption City, Ogun State, Nigeria \\ ${ }^{2}$ Department of Statistics, University of Ilorin, Nigeria
}

\begin{abstract}
Introduction: One of the greatest causes of morbidity and mortality in the Sub-Saharan Africa, particularly among young adults, is HIV/AIDS. Many mathematical models have been suggested for describing the epidemiology as well as the epidemiological consequences of the epidemic. A review of some these models would aid researchers in applying them to better understand and control the incidence and distribution of the disease in their countries.

Methodology: This study reviews some of the models proposed by various authors for describing the epidemiology as well as the epidemiological consequences of the HIV/AIDS epidemic and how some of them could be modified to suit the situations in other countries. We also discuss the limitations and the place of such models in the fight against the HIV epidemic.

Results: A clear explanation of the premises and assumptions on which the models were based was reached by reviewing the models across different scenarios.

Conclusion: Mathematical models have been very useful in HIV research, particularly for empirical studies on people living with HIV/AIDS (PLWHA). These models make predictions that generate questions of social and ethical interest.
\end{abstract}

Key words: HIV transmission; susceptible; infectious; epidemiology; homosexual; parameters; heterosexual

J Infect Dev Ctries 2010; 4(10):597-608.

(Received 29 September 2009- Accepted 9 June 2010)

Copyright $\odot 2010$ Akpa and Oyejola. This is an open-access article distributed under the Creative Commons Attribution License, which permits unrestricted use, distribution, and reproduction in any medium, provided the original work is properly cited.

\section{Introduction}

HIV transmission models aim to describe the dynamics of the spread of the disease by a system of equations in which the transition rates between defined states are specified quantitatively [1]. These models are complex and incorporate biological and behavioural variables which describe HIV transmission and its natural history to simulate the infection and disease process; therefore they could be used to reliably project HIV infection rates and AIDS cases if the model is shown to be valid and the data sets used are accurate [2]. Early examples of these types of models were reviewed by Isham [3].

This paper reviews various mathematical models already proposed in the context of HIV transmission and the AIDS epidemic. Emphasis is placed on the various forms of HIV transmission models and the assumptions under which the models were based. We also trace how transmission models evolved from the simplest population of homosexuals with homogeneity with respect to susceptibility, infectiousness and sexual mixing.

\section{Approaches to HIV/AIDS modeling}

Four different types of basic modeling approaches can be used to develop mathematical models for HIV/AIDS and some other infectious diseases: The deterministic models, the stochastic models, the statistical models (direct extrapolation and the state back-calculation method), and the Space-Kalman filter models [4].

This study focuses on deterministic models. This approach to modeling HIV/AIDS uses HIV transmission dynamics models which include the progression to AIDS and often have the population divided into compartments consisting of those who are susceptible, in each of the infection stages, or in the AIDS phase. In deterministic transmission models, the movement between these compartments by becoming infected, progressing to the next stage or AIDS, migrating, or dying is specified by systems of difference or differential equations [5].

Dynamic models and computer simulations are experimental tools for comparing regions or risk groups, testing theories, assessing quantitative 
conjectures, and answering questions [5]. They can also be used to theoretically evaluate, compare or optimize various detection, prevention, intervention, or control programs. Determistic models assume that all response variables, such as the numbers of susceptible people, infected people, and AIDS cases in the HIV epidemics, are deterministic functions of time ignoring completely the randomness of these variables and the randomness of all risk factors. The equations used in deterministic models are derived by taking into account the biological and the epidemiological as well as the clinical aspects of the HIV epidemics. By analyzing these equations, one may then study the behaviour and progression of the HIV epidemics as time progresses [4].

Some HIV transmission dynamics models are stochastic, with probabilities of moving to the next stage at each time step. Stochastic models assume that the response variables are a family of random variables indexed by time so that the HIV epidemic is a stochastic process [4]. Since nature is basically stochastic and many variables are subject to stochastic variations, stochastic models are more realistic than deterministic models [4]. However, the mathematics in stochastic models is usually more complicated and difficult than those involved in deterministic models. Under some very special conditions, results of the deterministic models may provide a close approximation to the results of the mean number of the stochastic models.

\section{Deterministic and stochastic models}

In HIV modeling for instance, deterministic models assume that response variables such as the numbers of susceptible individuals at time $t, S(t)$, infected people at time $t, I(t)$ and those developing AIDS at time $t, A(t)$ are deterministic functions of time. The model takes no account of the randomness of these variables or that of all risk factors [4]. Sometimes called compartmental models, deterministic models attempt to approximate what happens on the average at the population scale and therefore requires less data. Deterministic models are often described by a system of differences or differential or integral equations which are derived by taking into account the biological, epidemiological and clinical aspects of the disease.

Stochastic models, on the other hand, assume that the response variables are a family of random variables indexed by time so that the epidemic is basically a stochastic process [4]. Stochastic epidemic models are useful for small populations, possibly of isolated communities in which the known heterogeneity inherent in the population is of importance. As a result, the mathematics in stochastic models are usually more complicated and difficult than those involved in deterministic models [4] and do not lend easy explanation to the dynamics of the epidemic.

Deterministic models often (under some conditions) provide approximation to the behaviour of the stochastic mean and are consequently referred to as special cases of stochastic models. Epidemiologists find it more convenient to use compartmental models (see Figures 1 and 2) in which individuals are categorized into different disease progress stages (sub-groups or compartments). In HIV/AIDS, for instance, individuals are classified into (without complicated thought) three compartments designated as susceptible, infectious and recovered/immunized (SIR), which in the case of HIV is the group of those developing AIDS at a given time [4].

Fundamentals of deterministic epidemic transmission models; application to HIV/AIDS infection

Haberman [6] presented an excellent review of the basic approach to deterministic epidemic transmission models. We begin with simple models for the spread of HIV infection within a closed group of male homosexuals. The model is then made more realistic and complex by allowing for immunity from infection; allowing for an open population with migration and deaths being incorporated; allowing for variations in the progression of the infection ; and for variations in the population according to risk behaviour or other important characteristics, such as geographic region. It would have the effect of overstating the number of HIV infections, because the model would fail to take into account the fact that, to some degree, the epidemic may be limited or contained within a subgroup.

The models may be adapted to deal with heterosexual spread in a two-sex population and with needle sharing associated with IV-drug abuse [6]. We start with a simple model for the spread of HIV infection within a closed male homosexual community and assume that the total population has a fixed size, $\mathrm{n}$. 
We use the following notation:

$t=$ time

$S(t)=$ number of susceptible individuals at time $\mathrm{t}$

$I(t)=$ number of infected individuals without AIDS

at time $\mathrm{t}$

$A(t)=$ number of AIDS cases at time $\mathrm{t}$

$A C(t)=$ accumulated number of AIDS cases up to

time $\mathrm{t}$

$\gamma=$ rate of developing AIDS for infected individuals

$\beta=$ probability of infection from a sexual contact

with an infected individual.

$C=$ average number of contacts between sexual partners, and

$r=$ average number of new sexual partners per year

Suppose that susceptibles become infected through sexual contacts with partners, whom they choose randomly at a fixed rate from the community. If $N(t)$ is the number at risk of being chosen in this way, then two extreme values for $N(t)$ would be $N(t)=n$, the whole population, or $N(t)=I(t)+S(t)$, if each individual who develops AIDS is withdrawn from the class of infectives. We shall take the latter case as representing a reasonable approximation to reality; then a deterministic approximation to the underlying stochastic process governing the behaviour of $S(t), I(t)$ and $A(t)$ is provided by the following set of ordinary differential equations:

$$
\left.\begin{array}{l}
\frac{d S(t)}{d t}=-\lambda(t) S(t) \\
\frac{d I(t)}{d t}=\lambda(t) S(t)-\gamma I(t) \\
\frac{d A(t)}{d t}=\gamma I(t)
\end{array}\right\} \quad 1
$$

Where $\lambda(t)=\beta C r \frac{I(t)}{N(t)}$ and $N(t)=S(t)+I(t), \gamma$ can be interpreted as the parameter for an exponentially distributed random variable representing the lengths of time that infected individuals remain infective. The behaviour of the epidemic in the early stages when $S(t) \approx N(t)$ is given by $I(t) \approx I(0) \exp ((\beta C r-\gamma) t)$ and $t_{d}$, the doubling time is given by

$$
\begin{aligned}
& t_{d} \cong \frac{\log _{e} 2}{(\beta C r-\gamma)} \\
& R=\frac{\beta C r}{\gamma} \text { susceptibles. }
\end{aligned}
$$

$\mathrm{R}$ is the reproductive rate of the infection and must satisfy $R>1$ if an epidemic is to develop.

A more general model separates the infectives I into two classes, according to whether or not they ultimately develop AIDS. This allows for the possibility that the mean incubation period for AIDS $\gamma_{1}^{-1}$ is different from the mean infectious period among those seropositives who do not develop AIDS $\gamma_{2}^{-1}$. In the extreme case $\gamma_{2}=0$, so that the seropositives remain infectious indefinitely, as investigated by Bailey and Estreichar [7]. We now modify the notation, so that $\mathrm{I}_{1}, \mathrm{I}_{2}, \mathrm{~A}_{1}, \mathrm{~A}_{2}$ denote the numbers of infected individuals who will ultimately develop AIDS; the number of infectives who do not develop AIDS; the number of AIDS cases; and the number of non-infectious seropositives, respectively. Then the ordinary differential equations become

$$
\begin{aligned}
& \frac{d S(t)}{d t}=-\lambda(t) S(t) \\
& \frac{d I_{1}(t)}{d t}=\rho \lambda(t) S(t)-\gamma_{1} I_{1}(t) \\
& \frac{d I_{2}(t)}{d t}=(1-\rho) \lambda(t) S(t)-\gamma_{2} I_{2}(t) \\
& \frac{d A_{1}(t)}{d t}=\gamma_{1} I_{1}(t) \\
& \frac{d A_{2}(t)}{d t}=\gamma_{2} I_{2}(t)
\end{aligned}
$$

Where $I(t)=I_{1}(t)+I_{2}(t)$ is the total number of infectives at time t, $\rho$ is the probability that an individual enters the class of potential AIDS patients on withdrawal from the class of infectives, and a suitable choice for $N(t)$ in the definition of $\lambda(t)$ might be as an equation (1), or with

$$
N(t)=S(t)+I_{1}(t)+I_{2}(t)+A_{2}(t)=n-A_{1}(t) 4
$$


If $\gamma_{1}=\gamma_{2}$ then equation 2.49 reduces to 2.48 , and for this model, the overall reproductive rate of HIV infection is given by

$$
R=\left(\frac{\rho}{\gamma_{1}}+\frac{\rho}{\gamma_{2}}\right) \quad 5
$$

The model represented by equation (8) has been studied numerically by several authors [9-11].

\section{Allowing for migration in HIV transmission models}

So far, the model has been applied to a closed population. In order to apply the model to time periods beyond the initial stages of the spread of infection, it is necessary to allow immigration to the class of susceptibles and deaths from all classes. Suppose:

$m(t)=$ rate of migration to the class of susceptibles at time $\mathrm{t}$

$\mu=$ death rate of individuals without AIDS (in the form of the force of mortality)

$\sigma=$ extra death rate of individuals with AIDS (with $\sigma>\mu)$.

Then the differential equations 2.49 are modified as follows:

$$
\begin{aligned}
& \frac{d S(t)}{d t}=m(t)-\lambda(t) S(t) \\
& \frac{d I_{1}(t)}{d t}=\rho \lambda(t) S(t)-\left(\mu+\gamma_{1}\right) I_{1}(t) \\
& \frac{d I_{2}(t)}{d t}=(1-\rho) \lambda(t) S(t)-\left(\mu+\gamma_{2}\right) I_{2}(t) \\
& \frac{d A_{1}(t)}{d t}=\gamma_{1} I_{1}(t)-(\mu+\sigma) A_{1}(t) \\
& \frac{d A_{2}(t)}{d t}=\gamma_{2} I_{2}(t)-\mu A_{2}(t) \\
& \frac{d A C_{1}(t)}{d t}=\gamma_{1} I_{1}(t) \\
& \frac{d A C_{2}(t)}{d t}=\gamma_{2} I_{2}(t)
\end{aligned}
$$

where $N(t)=S(t)+I_{1}(t)+I_{2}(t)+A_{1}(t)+A_{2}(t)$ in the definition of $\lambda(t)$. This model has been presented and analyzed by many authors for the spread of various sexually transmitted diseases, and has been studied numerically by Anderson and May (1986) [9] and by Anderson et al. (1986) [10] using the simple form $\gamma_{1}=\gamma_{2}$ and $N(t)=S(t)+I_{1}(t)+I_{2}(t)+A_{1}(t)+A_{2}(t) . \quad$ This model has also been studied by Hyman and Stanley [12], using the form $m=\mu S_{0}$ where $S_{0}$ is the population size before the AIDS virus was introduced (so that there is a balance between flows into and out of the population) and $\rho=1$ (so that $I_{2}(t) \equiv 0$ and $\left.A_{2}(t) \equiv 0\right)$. Similarly, Thompson [13] explores numerically $\rho=1$ and $m(t)=m$, but does not test the model for goodness of fit against observed data.

An analytic solution has been found by Birkhead [14] under certain further restrictive assumptions. First, it is assumed that $N(t)=S(t)+I_{1}(t)$, so that not only the AIDS patient but also the non-infectious seropositives are excluded. Secondly, it is assumed that the immigration of susceptibles is at a rate proportional to $N(t)$ i.e. $m=m_{0} N(t)$, rather than being constant. Neither modification will be significant in the initial stages of the epidemic, but as the epidemic progresses it is plausible that changing behaviour could result in a reduced level of immigration into the homogeneously mixing male homosexual community being modeled. Then, explicit analytic formulae for $I(t)$ and $N(t)$ can be derived.

Birkhead [14] used a different interaction of $\rho:$ a proportion of the seropositives are assumed to develop full-blown AIDS and then cease sexual mixing, with the rest remaining infective and sexually active. Thus, the equation for $I_{1}(t)$ becomes

$$
\begin{aligned}
& \frac{d I_{1}(t)}{d t}=\lambda_{1}(t) S(t)-\left(\rho \gamma_{1}+\mu\right) I_{1}(t) \\
& \text { where } \\
& \lambda_{1}=\frac{\beta C r I_{1}(t)}{\left(S(t)+I_{1}(t)\right.}
\end{aligned}
$$

Equation (3) can be modified to model purely heterosexual spreading, by splitting the population according to sex and including partnership balance relationships. These balances are necessary to take account of situations where there are not enough women, so that men cannot have as many partners as they might like, and vice versa.

Other modifications have been introduced to the above basic model by Van Druten et al. [15], to allow 
for the fact that, at the start of the spread of infection in the population, few of its members are at risk and that the size of the population at risk should, therefore, be a dynamic variable. The effect of modeling the risk population in this way is to slow down the initial exponential rate of increase in the number of infectives (relative to the models described earlier), with most effect when the average number of sexual partners per infective is small (this parameter is proportional both to $r$ and the mean duration of a partnership and, for a fixed value of $r$, would be small when partnerships are short-lived.

Deterministic transmission models for HIV infections

Cooper [16] considered the spread of HIV within a population in Sub-Saharan Africa. Starting with a very simple model for homosexually transmitted HIV, Cooper divided the total host population into five categories of individuals: susceptibles, $X(t)$; infectives of types 1 (who go onto develop AIDS) and 2 (who eventually recover), $Y_{1}(t)$ and $Y_{2}(t)$ respectively; those with clinical AIDS, $A(t)$; and recovered non infectious individuals, $Z(t)$. Out of those infected, a proportion $f$ are of type 1 and develop AIDS at a rate of $v_{1}$; the remaining fraction $1-f$ are of type 2 and move into the non infections state at a rate of $v_{2}$. The final parameters model births and deaths: a constant rate $B$ of new susceptibles enter the population, there is a constant mortality rate $\mu$, and an additional death rate for those with clinical AIDS given by $\alpha$. Equation (8) below describes this model:

$$
\begin{aligned}
& \frac{d X}{d t}=B-(\mu+\lambda) X \\
& \frac{d Y_{1}}{d t}=f \lambda X-\left(\mu+v_{1}\right) Y_{1} \\
& \frac{d Y_{2}}{d t}=(1-f) \lambda X-\left(\mu+v_{2}\right) Y_{2} \\
& \frac{d A}{d t}=v_{1} Y_{1}-(\mu+\alpha) A \\
& \frac{d Z}{d t}=v_{2}-\mu Z
\end{aligned}
$$

The force of infections $\lambda$, in these equations, is given by $\lambda=c\left(\beta_{1} Y_{1}+\beta_{2} Y_{2}\right) / N$.
Here $\beta_{k}$ is the probability that infection will be acquired from an infected sexual partner of type k, and $Y_{k} / N$ is the probability that a randomly chosen partner will be an infective of type $k\left(N=X+Y_{1}+Y_{2}+A+Z\right)$. For simplicity, the transmission probability is per partnership rather than per sexual act. Again, it was also assumed that there is homogeneity in the sexual habits within the population.

In practically any community of non trivial size, there is a large variation in sexual habits, with different individuals having different numbers of sexual partners at any given time. Using subscript $i$ to denote the number of susceptibles having an average $i$ sexual partners per unit time (we split the population into a few discrete classes of sexual activity rather than have a more continuous range), equation (8) then becomes

$$
\begin{aligned}
& \frac{d X}{d t}=B_{i}-\left(\mu+\lambda_{i}\right) X_{i} \\
& \frac{d Y_{1 i}}{d t}=f \lambda_{i} X_{i}-\left(\mu+v_{1}\right) Y_{1 i} \\
& \frac{d Y_{2 i}}{d t}=(1-f) \lambda_{i} X_{i}-\left(\mu+v_{2}\right) Y_{2 i} \\
& \frac{d A_{i}}{d t}=v_{1} Y_{1 i}-(\mu+\alpha) A_{i} \\
& \frac{d Z_{i}}{d t}=v_{2}-\mu Z_{i}
\end{aligned}
$$

The force of infection for susceptibles in the ith group is assumed to be proportional to the average number of partners per unit time $i$, so $\lambda_{i}=i \lambda$ where $\lambda$ is the probability that a randomly chosen partner will produce infection and is given by

$$
\lambda=\frac{\sum_{i} i\left(\beta_{1} Y_{1 i}+\beta_{2} Y_{2 i}\right)}{\sum_{i} i N_{i}}
$$

$\lambda$ depends on the probability that a given partner is infectious, and the probability $\beta_{k}$ that an infectious partners infects a susceptible. The factors of $i$ in the expression for $\lambda$ weight potential partners by their degree of sexual activity, since a highly promiscuous individual is more likely to be available as a potential partner. 
Deterministic models for heterosexual transmission of HIV infections

One of the unique features of a heterosexual transmission is the fact that the population consists of two sets of individuals, male and female, with the male being infected by an infectious female and vice versa. Cooper [16] extended the model in equation (8) to incorporate a simple model of HIV transmission within a closed heterosexual population that is heterogeneous with respect to sexual activity rates. Denoting terms relating to females with a prime, leaving the activity groups as before, and assuming that the progress of the disease is the same in males and females (both use the same parameter $v$ ), the model is given as

$$
\left.\begin{array}{rl}
\frac{d X_{i}}{d t} & =-\lambda_{i} X \\
\frac{d Y_{i}}{d t} & =\lambda_{i} X_{i}-v Y_{i} \\
\frac{d X_{i}^{\prime}}{d t} & =-\lambda_{i}^{\prime} X^{\prime} \\
\frac{d Y_{i}^{\prime}}{d t} & =\lambda_{i}^{\prime} X_{i}^{\prime}-v Y^{\prime}
\end{array}\right\}
$$

As with the homosexual model, $\lambda_{i}=i \lambda$ where $\lambda$ represents the probability that any (randomly chosen) female partner will infect a susceptible male, and vice versa for $\lambda^{\prime}$. Analogously to the homosexual case, noting that males are only infected by females and females by males, these probabilities are given by

$$
\lambda=\beta^{\prime} \frac{\sum_{i} i Y_{i}^{\prime}}{\sum_{i} i N_{i}^{\prime}} \text { and } \lambda^{\prime}=\beta \frac{\sum_{i} i Y_{i}}{\sum_{i} i N_{i}} .
$$

One of the major criticisms against this type of model (and also the earlier ones) is that it assumes no correlation between individuals in the various classes of sexual activities in their search for partners. In reality we would expect those more promiscuous individuals to seek out partners of like mind, and vice versa [16].

The simple model in equation (11) lacks several features of the earlier homosexual models, namely births, deaths, and a class of individuals with AIDS.
Cooper [16] extended this model to include births, deaths and a class of individuals with AIDS. The equations describing the extended model are shown below:

$$
\left.\begin{array}{c}
\frac{d X_{i}}{d t}=B_{i}-\left(\mu+\lambda_{i}\right) X_{i} \\
\frac{d Y_{i}}{d t}=\lambda_{i} X_{i}-(\mu+v) Y_{i} \\
\frac{d A_{i}}{d t}=v Y_{i}-(\mu+\alpha) A_{i} \\
\frac{d X_{i}^{\prime}}{d t}=B_{i}-\left(\mu+\lambda_{i}^{\prime}\right) X_{i}^{\prime} \\
\frac{d Y_{i}^{\prime}}{d t}=\lambda_{i}^{\prime} X_{i}^{\prime}-(\mu+v) Y_{i}^{\prime} \\
\frac{d A_{i}^{\prime}}{d t}=v Y_{i}^{\prime}-(\mu+\alpha) A_{i}^{\prime}
\end{array}\right\}
$$

As before, variables with a prime denote females, and those without denote males.

Garnett and Anderson [17] presented a description of the development and analysis of a mathematical model of the spread and demographic impact of HIV in heterosexual communities in developing countries. The model extended previous work in this area by the representation of patterns of mixing between and within different age and sexual activity classes in a two-sex structure. Summary parameters were derived to represent different mixing patterns, ranging from assortative via random to disassortative, as were methods to ensure that particular mixing patterns between different age and sexual classes (stratified on the basis of rates of sexual partner change) met constraints that balanced the supply and demand for sexual partners, as AIDSinduced mortality influences the demographic structure of a population.

The model consists of a system of partial differential equations to describe changes in the numbers of susceptibles ( $X$ ) of infected individuals $(Y)$, and of individuals with $\operatorname{AIDS}(A)$ of both sexes, with respect to time $t$ and age $a$. It has the following structure: 


$$
\begin{aligned}
& \frac{\partial X_{k l}(a, t)}{\partial a}+\frac{\partial X_{k l}(a, t)}{\partial t}=-\left[\lambda_{k l}(a, t)+\mu_{k}(a)\right] X_{k l}(a, t) \\
& \frac{\partial Y_{1 k l}(a, t)}{\partial a}+\frac{\partial Y_{1 k l}(a, t)}{\partial t}=\lambda_{1 k l}(a, t) X_{k l}(a, t)-\left[\gamma_{1}(a)+\mu_{k}(a)\right] Y_{1 k l}(a, t) \\
& \frac{\partial Y_{s k l}(a, t)}{\partial a}+\frac{\partial Y_{s k l}(a, t)}{\partial t}=\gamma_{(s-1)}(a) Y_{(s-1) k l}(a, t)-\left[\gamma_{s}(a)+\mu_{k}(a)\right] Y_{s k l}(a, t) \\
& \frac{\partial A_{k l}(a, t)}{\partial a}+\frac{\partial A_{k l}(a, t)}{\partial t}=\gamma_{3}(a) Y_{3 k l}(a, t)-\left[\mu_{k}(a)+\alpha(a)\right] A_{k l}(a, t)
\end{aligned}
$$

where $X_{k l}(a, t)$ denotes the number of susceptible individuals of sex $k$, sexual activity group $l$, and age $a$ at time $t$. Sexual activity groups are defined on the basis of rates of sexual partner change per unit of time ( $\mathrm{n}$ in total). Once infected, individuals pass via three states $(s=1,2$ and 3$)$ to denote disease progression prior to developing AIDS. This division of the infected but non AIDS variable $Y$ into three categories is made to mirror variable infectiousness over the incubation period of AIDS [8], with patients moving from a state of high infectiousness, via a state of low infectiousness, back to a state of high infectiousness just prior to the development of AIDS. The other subscript labels on $Y_{s k l}(a, t)$ are as defined for $X_{k l}(a, t)$ and they also apply for the AIDS compartment, $A_{k l}(a, t)$. The total population at time $t, N(t)$ is given by

$$
N(t)=\sum_{k=1}^{2} \sum_{l=1}^{n}\left[\int_{0}^{\infty}\left(X_{k l}(a, t)+\sum_{s=1}^{3} Y_{s k l}(a, t)+A_{k l}(a, t) d a\right] 14\right.
$$

Garnett and Anderson [17] assumed that the flow of individuals from $Y$ to $A$ is based on the assumption that all who acquire infection eventually develop AIDS. It is possible that some small fraction of infected individuals may not progress to AIDS within their natural lifespan but the magnitude of this proportion is unknown.

Susceptibles acquire HIV infection at sex-, sexual activity group-, age- and time-dependent rates and enter the first stage of infection $Y_{s k l}(a, t)$ where $s=1$. They pass through the three stages of infection at a stage- and age-dependent rate $\gamma_{s}(a)$. Individuals are removed from the population at an age- and sexdependent rate per capital mortality rate $\mu_{k}(a)$ (per year), and those who have AIDS also have an additional age-dependent mortality rate $\alpha(a)$ (per year).

Further details and analyses of these models are obtainable from Garnett and Anderson [17]. The principal conclusion of the study was that the pattern of mixing between age and sexual activity classes, combined with the assumptions made to balance supply and demand between the sexes, have a major influence on the predicted pattern of HIV spread and the demographic impact of AIDS.

A data-based mathematical model to access the epidemiological consequences of heterosexual, intravenous drug use and perinatal transmission in New York City was suggested by Blower et al. [18]. The results demonstrated the significance of the dynamic interaction. Scenario analysis of the model was used to suggest a new explanation for the stabilization of the seroprevalence level that has been observed in the New York City intravenous drug use community; the proposed explanation does not rely upon any intravenous drug use or sexual behavioural changes. Gender specific risks of heterosexual transmission in intravenous drug users were also explored by scenario analysis. The model was used to predict future numbers of adult and paediatric AIDS cases; a sensitivity analysis of the model showed that the confidence intervals on these estimates were extremely wide. This prediction variability was due to the uncertainty in estimating the values of the thirty variables of the model. However, the sensitivity analysis reveals that only a few key variables were significant in contributing to the AIDS case prediction variability. Partial rank correlation coefficients were calculated and used to identify and to rank the importance of these key variables.

The model divided the population of women into the categories of susceptible individuals, infected individuals, and individuals with clinical AIDS, with each category divided into five subgroups. The rate of change of the population sizes of the five subgroups of susceptible women are given below: 


$$
\begin{aligned}
& \frac{d X_{1 f}}{d t}=-X_{1 f} \alpha_{f} \lambda_{i}-X_{1 f} a_{d f} \\
& \frac{d X_{2 f}}{d t}=-X_{2 f} \beta_{f} \lambda_{j}-X_{2 f} a_{d f} \\
& \frac{d X_{3 f}}{d t}=-X_{3 f}\left(\alpha_{f} \lambda_{i}+C_{f s}(t) \lambda_{3 f}\right)-X_{3 f} a_{d f} \\
& \frac{d X_{4 f}}{d t}=-X_{4 f}\left(\beta_{f} \lambda_{j}+C_{f b}(t) \lambda_{4 f}\right)-X_{4 f} a_{d f} \\
& \frac{d X_{5 f}}{d t}=-X_{5 f} C_{f n}(t) \lambda_{s f}-X_{5 f} a_{f}
\end{aligned}
$$

where the variables $X_{1 f}, X_{2 f}, X_{3 f}, X_{4 f}$ and $X_{5 f}$ are numbers of susceptible women who have the single intravenous drug use (IVDU) risk factor of sharing needles with strangers (stranger user); those who have the single IDVU risk factors of sharing needles with friends (buddy user); those who have dual risk factors (sexually active stranger users); those who have dual risk factors (sexually active buddy users); and those who have the single risk factor through heterosexual transmission respectively; and where $\alpha_{f}$ is the rate of sharing needles per female; $\lambda_{i}$ is the female stranger user intravenous drug use transmission probability; $a_{d f}$ is the non HIV mortality rate of intravenous drug users; $\beta_{f}$ is the rate of change of drug buddies per female; $\lambda_{j}$ is the female buddy users intravenous drug use transmission probability; $C_{f s}$ is the rate of change of sex partners per female stranger user; $\lambda_{3 f}$ is the probability of acquiring HIV from heterosexual transmission per female stranger user; $C_{f b}$ is the rate of change of sex partners per female buddy user; $\lambda_{4 f}$ is the probability of acquiring HIV from heterosexual transmission per female buddy users; $C_{f n}$ is the rate of change of sex partners per female non intravenous drug users; $\lambda_{5 f}$ is the probability of acquiring HIV from heterosexual transmission per female non intravenous drug user; and $a_{f}$ is the non HIV mortality rate.

Furthermore, let $Y_{1 f}, Y_{2 f}, Y_{3 f}, Y_{4 f}$ and $Y_{5 f}$ denote the numbers of infected women corresponding to the five subgroup. Additionally, $A_{1 f}, A_{2 f}, A_{3 f}, A_{4 f}$ and $A_{5 f}$ denote the numbers of women with AIDS in the corresponding five subgroups. Then the rates of change of the population sizes of the five subgroups of infected women are expressed as

$$
\begin{aligned}
& \frac{d Y_{1 f}}{d t}=X_{1 f} \alpha_{f} \lambda_{i}-\left(1 / v_{a}\right) Y_{1 f}-Y_{1 f} a_{d f} \\
& \frac{d Y_{2 f}}{d t}=X_{2 f} \beta_{f} \lambda_{j}-\left(1 / v_{a}\right) Y_{2 f}-Y_{2 f} a_{d f} \\
& \frac{d Y_{3 f}}{d t}=X_{3 f}\left(\alpha_{f} \lambda_{i}+C_{f s}(t) \lambda_{3 f}\right)-\left(1 / v_{a}\right) Y_{3 f}-Y_{3 f} a_{d f} \\
& \frac{d Y_{4 f}}{d t}=X_{4 f}\left(\beta_{f} \lambda_{j}+C_{f b}(t) \lambda_{4 f}\right)-\left(1 / v_{a}\right) Y_{4 f}-Y_{4 f} a_{d f} \\
& \frac{d Y_{5 f}}{d t}=X_{5 f} C_{f n}(t) \lambda_{s f}-\left(1 / v_{a}\right) Y_{5 f}-Y_{5 f} a_{f}
\end{aligned}
$$


where $v_{a}$ is the average duration of stay in the infected class.

Similarly, the rates of change of the population sizes of the five subgroups of women with AIDS are given below:

$$
\left.\begin{array}{l}
\frac{d A_{1 f}}{d t}=\left(1 / v_{a}\right) Y_{1 f}-A_{1 f}\left(1 / s_{a}\right)-A_{1 f} a_{d f} \\
\frac{d A_{2 f}}{d t}=\left(1 / v_{a}\right) Y_{2 f}-A_{2 f}\left(1 / s_{a}\right)-A_{2 f} a_{d f} \\
\frac{d A_{3 f}}{d t}=\left(1 / v_{a}\right) Y_{3 f}-A_{3 f}\left(1 / s_{a}\right)-A_{3 f} a_{d f} \\
\frac{d A_{4 f}}{d t}=\left(1 / v_{a}\right) Y_{4 f}-A_{4 f}\left(1 / s_{a}\right)-A_{4 f} a_{d f} \\
\frac{d A_{5 f}}{d t}=\left(1 / v_{a}\right) Y_{5 f}-A_{5 f}\left(1 / s_{a}\right)-A_{5 f} a_{f}
\end{array}\right\}
$$

where $s_{a}$ is the average survival time from diagnosis of AIDS to death.

The model also includes fifteen corresponding equations for males. These equations have the same structure as the equation for females but contain male specific values for intravenous drug use and sexual values for intravenous drug use and sexual behaviour parameters.

Williams and Anderson [19] formulated a model (for HIV-1 in England and Wales) which mimics transmission within and between different sexual activity classes (or needle-sharing classes in the case of intravenous drug users) and within and between different risk groups, such as male homosexuals, intravenous drug users, and heterosexuals. Patterns of mixing and sexual contact are described by mixing matrices whose elements define the degree of assortative (like with like) or disassortative (like with unlike) contact between different stratifications of the sexually active population. The model is a deterministic compartmental model of the type outlined by Anderson and May [8], where the population is not age structural but is stratified into five compartments representing an isolated population of sexually active adults, with a recruitment rate equal to the rate of leaving the population (due to cessation in sexual activity or natural mortality), so, in the absence of HIV, the population size is constant. Each compartment corresponds to one epidemiological state: first an uninfected susceptible state $X$; then three consecutive stages of HIV incubation, $Y_{1}, Y_{2}, Y_{3}$; and finally A, the state of fully developed AIDS (there is no recovered class). The three incubation stages, which may be associated with differing degrees of infectiousness, correspond to the initial period of high HIV antigen concentration shortly after infection, the longer asymptomatic stage and the final stage with high levels of antigenaemia as the individual progresses to persistent generalized lymphadenopathy and AIDS-related complex before reaching fully developed AIDS [9,10,12,19].

There are two modes by which susceptibles may become infected: through sexual contact or by the sharing of needles. It was assumed that individuals in the AIDS compartment are neither sexually active nor do they share needles; these may be optimistic assumptions.

Each compartment is subdivided into heterosexual, IDU, and homosexual risk groups with each further subdivided into subgroups with low, medium, and high rates of acquisition of sexual partners. The proportion of new recruits allocated to each subgroup is held constant over time. To simplify the analysis, no distinction is made between males and females. This has the advantage of simplicity but, concomitantly, fails to allow for differing probabilities of the transmission of HIV from males to females and vice versa [19]. In essence, the labels heterosexual and homosexual are used to distinguish crudely between populations with differing HIV transmission rates, sexual mixing patterns, and distributions of activity levels (and associated rates of changing partners). The IDU group has a distinct set of parameters relating to sexual contact but is further distinguished by being subject to HIV transmission through the exchange of needle used for drug injections.

The model consists of a set of coupled ordinary differential equations with the following structure:

$$
\begin{aligned}
& \frac{d X_{g l t}}{d T}=\Lambda_{g l t}-X_{g l t}\left(\mu+\lambda_{g l t}\right) \\
& \frac{d Y_{1 g l t}}{d t}=X_{g l t} \lambda_{g l t}-Y_{1 g l t}\left(\mu+\gamma_{1}\right) \\
& \frac{d Y_{2 g l t}}{d t}=Y_{1 g l t} \gamma_{1}-Y_{2 g l t}\left(\mu+\gamma_{2}\right) \\
& \frac{d Y_{3 g l t}}{d t}=Y_{2 g l t} \gamma_{2}-Y_{3 g l t}\left(\mu+\gamma_{3}\right) \\
& \frac{d A_{g l t}}{d t}=Y_{3 g l t} \gamma_{3}-A_{g l t} \alpha
\end{aligned}
$$


where $\quad X, Y_{1}, Y_{2}, Y_{3}$, and $A \quad$ represent epidemiological states defined earlier. The activity groups (heterosexual, IDU or homosexual) are represented by subscript $g$, sexual activity by subscript $l$, and time by subscript $t$. $\Lambda$ represents the rate at which susceptible individuals join the sexually active population, $\mu$ the non AIDS mortality rate (that of leaving the sexually active population), and $\lambda$ the per capita force of infections. $\gamma_{1}, \gamma_{2}$ and $\gamma_{3}$ represent the rates of progression from each incubation stage, and $\alpha$ the AIDS-related mortality rate. The term for the rate of recruitment (omitting subscript $\mathrm{t}$ ) is defined as

$$
\Lambda_{g l}=p_{g l} v \sum_{h} \sum_{m}\left(X_{h m}+\sum_{k} Y_{k h m}\right)
$$

Here $p_{g l}$ represents the proportion of the original population in group $g$, and activity level $l, v$ represents the per capita recruitment rate, and $k$ the incubation stage; the summation simply gives the total non-AIDS population. The force or per capita rate of infection term $\lambda$ is the sum of the force of infection for sexual contact and that for needle sharing contact: with heterogeneous mixing that explains the mechanism of transmission of virus from FSW to adult males and vice versa was presented by Srinivasa Rao [20]. The male population that mixes with FSWs was divided into four classes: male susceptible, HIV infective, STD infective, and both STD and HIV infective. The rate of additions of male susceptibles from non-susceptible and STD cured were taken to be exponential, though in reality they may be different. At the same time, the number of individuals infected with HIV and STD per unit of time and the number who withdraw from risk behaviour are removed from the susceptibles. The withdrawal number was based on a general withdrawal rate, or dependent on individual behaviour. HIV positive individuals can still be STD infective and vice versa. Other withdrawal cases, e.g., natural deaths, change of risk behaviour, etc., either from HIV (STD) infective or from dual infected, can be removed from respective compartments. Let $\mathrm{F}_{10}$, $F_{20}, F_{21}$ and $F_{12}$ be four coefficients of movements from male susceptible to HIV infective, male susceptible to STD infective, HIV infective to both STD and HIV infective, respectively. Denote $\lambda_{m}$ as the entry rate into the male susceptible compartment, and $\gamma_{m}$ and $\gamma_{m}^{\prime}$ as recovery rates from STD

$$
\lambda_{g l}=\sum_{h} \sum_{m} \sum_{k}\left(c_{g l} \rho_{g l h m} \beta_{k g h}+\kappa_{g l} \rho_{N g l h m} \beta_{N k g h}\right) \frac{Y_{k h m}}{X_{h m}+\sum_{L} Y_{L h m}}
$$

Here $c$ and $k$ represent rates of acquisition of new sexual and needle-sharing patterns respectively; $g$ and $h$ define at-risk groups; $L$ and $m$ define sexual activity groups; $\rho_{g l h m}$ defines the proportions of sexual and needle-sharing partnerships that subgroup $g l$ has with subgroup $h m, ; k$ and $L$ represent incubation stages and $\beta_{k g h}$ and $\beta_{N k g h}$ denote probabilities of transmission (defined per partner) for sexual contacts and needle sharing.

Furthermore, a model based on the prevention of transmission to and from female sex workers (FSWs) representing without and with HIV infection. $\mu_{m}$ is mortality rate; $\beta_{10}$ is HIV transmission probability from FSW to a susceptible male; $\beta_{20}$ is STD transmission probability from FSW to a susceptible male; $\beta_{21}$ is HIV transmission probability from a FSW with both STD and HIV; and $\delta_{1}$ and $\delta_{2}$ are withdrawal rates of males due to reasons other than sexual activity. 
The model is given as

$$
\begin{aligned}
& \frac{d X_{0}}{d t}=\lambda_{m}-\left(F_{10}+F_{20}+\mu_{m}\right) X_{0}+\gamma_{m} X_{2} \\
& \frac{d X_{1}}{d t}=F_{10} X_{0}-\left(\mu_{m}+\delta_{1}+F_{21}\right) X_{1}+\gamma_{m}^{\prime} X_{3} \\
& \frac{d X_{2}}{d t}=F_{20} X_{0}-\left(\mu_{m}+\delta_{2}+F_{12}\right) X_{2}-\gamma_{m}^{\prime} X_{2} \\
& \frac{d X_{3}}{d t}=F_{21} X_{1}+F_{12} X_{2}-\left(\mu_{m}+\delta_{1}+\delta_{2}\right) X_{3}-\gamma_{m}^{\prime} X_{3}
\end{aligned}
$$

where

$$
F_{i j}=\beta_{i j} C\left[\frac{Y_{i}+Y_{j}}{\sum_{k=0}^{3} Y_{k}}\right]\{i=1,2 ; j=0,1,2\}
$$

impractical. Thus, modeling is essential for exploratory work. Apart from these important reasons for conducting mathematical modeling, repeatable experiments and accurate data are usually not available in epidemiology; hence mathematical and computer simulation models must be used to perform needed theoretical experiments with different parameter values and different data sets. It is easy in a computer simulation to determine what will happen if one or several parameters are changed [5].

Another very important reason for HIV modeling is the value of models for theoretical evaluation and comparisons of detection, prevention, therapy, and control programs. Epidemiologists and politicians need to understand the effects of different policy decisions on the dynamics of a particular infection/chronic disease in order to decide which approach is the most ethical, appropriate and economical [5].

However, as Hethcote and Van Ark [5] mentioned, some limitations of epidemic modeling, such as the fact that an epidemiological model is not reality but rather it is an extreme simplification of reality. Also, deterministic models do not reflect the role of chance in disease spread and do not provide confidence intervals on results. Stochastic models incorporate chance, but are usually harder to analyze than the corresponding deterministic model. 


\section{Acknowledgement}

We would like to thank the management of the Redeemer's University for providing access to research materials which enhanced the completion of this work.

\section{References}

1. De Angelis D, Day NE, Gill ON (1998) Acquired Immune Deficiency Syndrome Projections in England and Wales: Interplay of Methodology and Data. JR Statist Soc A 161: Part 2: 167-176.

2. Chin J and Lwanger S (1991) Estimation and Projection of Adult AIDS Cases: a Simple Epidemiological Model. Bulletin of WHO 69: 399-406.

3. Isham V (1988) Mathematical Modeling of the Transmission Dynamics of HIV Infection and AIDS: A Review. JR Stat Soc A 151: 5-30.

4. Wan-Yuan $T$ (2000) Stochastic Modeling of AIDS Epidemiology and HIV Pathogenesis. World Scientific Publishing Co. Pte. Ltd, Singapore, 431 p.

5. Hethcote HW and Van Ark JW (1992) Modeling HIV Transmission and AIDS in the United States. Lecture notes in Biomathematics 95. Springer-Verlag, New York, 234 p.

6. Haberman S (1990) Actuarial Review of Models for Describing and Predicting the Spread of HIV Infection and AIDS. JIA 117: 319-405.

7. Bailey NTJ and Estreichar J (1987) Epidemic Prediction and Public Health Control, with Special Reference to Influenza and AIDS. Proc. $1^{\text {st }}$ World Congress of Bernoulli Society (Tashkent, September 1986).

8. Anderson RM and May RM (1991) Infectious Diseases of Humans: Dynamics and Control. Oxford University Press, 768 p.

9. Anderson RM and May RM (1986) The Invasion, Persistence and Spread of Infectious Disease within Animal and Plant Communities. Phil Trans Roy Soc B 314: 533-570.

10. Anderson RM, Medley GF, May RM, Johnson AM. (1986) A Preliminary Study of the Transmission Dynamics of the Human Immunodeficiency Virus (HIV), the Causative Agent of AIDS. IMA J Math Appl Med and Biol 3: 229-263.

11. Blythe SP and Anderson RM (1988) Heterogeneous Sexual Activity Models of HIV Transmission in Male Homosexual Populations. IMA J Math Appl Med Biol D: 1-19.

12. Hyman JM and Stanley EA (1988) Using Mathematical Models to Understand the AIDS Epidemic. Mathematical Bioscience 90: 415-473.

13. Thompson JR (1987) AIDS: Old Disease, New Society. Technical Report 87-1, Department of Statistics, Rice University, Texas.

14. Birkhead BG (1987) A Mathematical Model of the Transmission of the HIV under Diminishing Recruitment-an Exact Solution. Department of Statistical Sciences, University College, London, Technical Note.

15. Van Druten JAM, De Boo TH, Reeintjes JC, Heisterkaamp SH, Coutinho RA, Bos JM, Ruitenberg EJ. (1887) Reconstruction and Prediction of Spread of HIV Infection in Populations of Homosexual Men. Proc. EC Workshop On Statistical Analysis And Mathematical Modeling of AIDS. Biethoven, December 1986. Oxford University Press: Oxford.

16. Cooper J (2004) Modeling the Spread of HIV in sub-Saharan Africa. (Online) Available At: Http://Newtou.Dtc.Ox.Ac.Uk/Cooper/Hiv-Africa.Pdf. Accessed 20/11/2008.

17. Garnett GP and Anderson RM (1993) Factors Controlling the Spread of HIV in Heterosexual Communities in Developing
Countries: Patterns of Mixing Between Different Age and Sexual Activity Classes. Phil Trans R Soc Lond B 342: 137-159.

18. Blower SM, Hartel D, Dowlatabadi H, Anderson RM, May RM (1991) Drugs, Sex and HIV: A Mathematical Model for New York City. Phil Trans R Soc Lond B 321: 171-187.

19. Williams JR and Anderson RM (1994) Mathematical Models of the Transmission Dynamics of Human Immunodeficiency Virus in England and Wales: Mixing between different risk Groups. JR Statist Soc A 157: 69-87.

20. Srinivasa Rao, ASR (2003) Mathematical Modeling of AIDS Epidemic in India. Current Science 84: 1192-1197.

21. Mukandavire Z and Garirar W (2007) Effect of Public Health Educational Campaigns and the Role of Sex Workers on the Spread of HIV/AIDS among Heterosexuals. Theoretical Population Biology 72: 346-365.

22. Abbas UL, Anderson RM and Mellors JW (2007) Potential Impact of Antiretroviral Chemoprophylaxis on HIV-1 Transmission in Resource-Limited Settings. PLOS ONE 2; E875 Doi: 10.1371 (1-11).

23. Sani A, Kroese DP, Pollet PK (2007) Stochastic Models for the Spread of HIV in a Mobile Heterosexual Population. Math Biosc 208: 98-124.

\section{Corresponding author}

Onoja Matthew Akpa

Department of Mathematical Science

Redeemer's University

PMB 3005

Redemption City, Ogun State, Nigeria

Mobile: +234 8032159579 .

Email: onojamatthew@yahoo.co.uk

Conflict of interests: No conflict of interests is declared. 